\title{
Galactic Rotation Effect and the Origin of Cosmic Radiation
}

\author{
M. S. VALLARTA \\ Instituto de Física, Universidad de México, México, D. F.
}

\begin{abstract}
A FUNDAMENTAL question related to primary cosmic radiation is whether the latter is a local or a universal phenomenon, i.e., whether it exists only in the immediate vicinity of the earth or all through the universe. This question has to do on the one hand with general cosmological problems and on the other with the problem of the origin of primary cosmic radiation. In the former case the total energy contained in the form of cosmic rays is only a very small fraction of the total energy of the universe, while in the latter it constitutes a relatively large fraction, certainly not less than $1 / 10,000$ part of the total energy, including the mass energy. This estimate may be readily made by taking into account the known density of primary rays in our vicinity and the average energy of each particle, assuming a power spectrum of the form $\left(K / E^{s}\right) d E$ with $s$ between 2 and 3. It is clear that in these two different cases the source of the cosmic radiation must be sought in quite different processes.

The suggestion that cosmic radiation exists throughout the universe has been put forward by several authors, among them Lemaître, ${ }^{1}$ Millikan, ${ }^{2}$ and Milne. ${ }^{3}$ According to the first, the cosmic rays are the product of the disintegration of the "primeval atom" where all the mass of the universe was originally contained, while the second assumes them to be the result of the annihilation of the most abundant elements constituting the universe, i.e., helium, oxygen, carbon, and silicon. Neither of these theories seems to have been worked out in detail quantitatively. According to the third, primary cosmic rays are a consequence of the motion of the observer in a world governed by the postulates of his kinematical relativity.

Recently Fermi ${ }^{4}$ on the one hand, and Alfvén, Richtmyer, and Teller ${ }^{5}$ on the other have suggested that primary cosmic radiation either is confined within our own galaxy or within a region of the order of dimensions of our planetary system. In Fermi's theory primary cosmic rays would be accelerated by the balancing of energy of interstellar or stellar matter acting inside of

${ }^{1}$ G. Lemaître, Nature 128, 704 (1931). L'atome primitif. (Génève, 1948).

${ }_{2}^{2}$ Millikan, Neher, and Pickering, Phys. Rev. 61, 397 (1942); 66, 295 (1944)

${ }^{3}$ E. A. Milne, Nature 135, 183 (1935).

${ }^{4} \mathrm{E}$. Fermi, unpublished, private communication to the author.

5 Alfvén, Richtmyer, and Teller, Phys. Rev. 75, 892 (1949).
\end{abstract}

the Milky Way and would exist essentially within the latter; in the theory suggested by Alfvén, Richtmyer, and Teller primary particles would derive their energy from electric fields arising from streams of particles shot out from the sun and would be trapped by the action of the sun's permanent magnetic field. Both theories have points in their favor.

The fundamental question is thus whether primary cosmic charged particles come from outside our galaxy or not. The purpose of this note is to point out that there is a way of deciding this question experimentally by measuring the galactic rotation effect. This is a first order relativistic effect arising from the fact that because of the rotation of our galaxy there is a relative velocity of the order of $300 \mathrm{~km} / \mathrm{sec}$. between the observer on the earth and the primary particle outside the galaxy. The existence of this effect was pointed out by Compton and Getting ${ }^{6}$ and its theory, taking into account the action of the earth's magnetic field for particles moving in the geomagnetic equator, was worked out by Vallarta, Graef, and Kusaka. ${ }^{7}$ The expected effect is a diurnal variation of the directional intensity depending on sidereal time, of amplitude of the order of 0.2 percent depending on the energy spectrum of the primaries, their low energy cut-off and the ratio between positives and negatives. The effect should decrease if there are equal numbers of positives and negatives. The experiment, as suggested, consists in measuring the vertical intensity at the geomagnetic equator over a sufficiently long period of time so as to ensure the proper statistical precision. If properly performed, and in view of the high probability that all primaries are positive, it should be in the nature of a crucial experiment.

Recent advances in counter technique, particularly as regards stability over long periods of time, ensure much better experimental conditions than seemed possible only a few years ago. An experiment as suggested, performed with proper precautions throughout, would seem to have a good chance of success. Dr. Vikram Sarabhai of Ahmedabad, India, has offered to try it at Kodaikanal in Southern India, almost on the geomagnetic equator. His results will be awaited with very considerable interest.

\footnotetext{
${ }^{6}$ A. H. Compton and I. A. Getting, Phys. Rev. 47, 817 (1935).

${ }^{7}$ Vallarta, Graef, and Kusaka, Phys. Rev. 55, 1 (1939).
} 